\title{
Streptococcal skin infection and acute glomerulonephritis
}

\author{
Hugh C. Dillon, JR \\ M.D. \\ Professor of Pediatrics and Associate Professor of Microbiology, \\ University of Alabama in Birmingham, Birmingham, Alabama 35233
}

\section{Introduction}

The role of streptococcal skin and soft tissue infections in the development of acute glomerulonephritis (AGN) is now clearly established. Among the early studies comparing the epidemiology of AGN and rheumatic fever it was noted that the former complication often followed deep or suppurative streptococcal infection, including wound infection, whereas rheumatic fever was commonly associated only with superficial pharyngeal infections. Impetigo, erisipelas and wound infections were all incriminated as antecedent infections of AGN in early studies (Dillon, 1967). Furthermore, as recently postulated (Wannamaker, 1970), the socalled 'trench nephritis', common during World War I, probably represented post-streptococcal AGN, with streptococcal skin or wound infection being of primary aetiologic importance.

Interest in the role of skin infection in AGN appears to have been relatively dormant during the period of World War II and the immediate post-war era. In contrast, intensive interest in streptococcal pharyngeal infection was evident and it was during this period that the relation between streptococcal pharyngitis and acute rheumatic fever became unequivocally established (Rammelkamp, Denny \& Wannamaker, 1952). Shortly thereafter the now classic studies of epidemic AGN following throat infection with streptococcal type M-12 led to the establishment of the concept of there being 'nephritogenic' strains of streptococci (Rammelkamp \& Weaver, 1953). It soon became apparent from studies in widely different areas that limited serotypes of group A streptococci, notably M-types 1 and 12, accounted for most cases of AGN following streptococcal sore throat (Wannamaker, 1967).

Following the observations that led to the hypothesis of there being nephritogenic streptococci, epidemiologic studies of impetigo in Great Britain (Parker, Tomlinson \& Williams, 1955) suggested that limited serotypes of streptococci, most of which were typable at that time only by slide agglutination serotyping (which identifies T-antigens, see below) accounted for most cases of impetigo. These investigators coined the term 'impetigo streptococci'. AGN was not reported in that particular investigation but it has become subsequently apparent that these studies represented a fundamental contribution to the understanding of differences in the epidemiology of streptococcal skin and pharyngeal infections. Sporadic reports of AGN following impetigo, most of which were from the south eastern area of the United States where impetigo has long been recognized to be a perennial problem during the warm and humid months of summer and early autumn, were notable in that the streptococci isolated from these patients were not further identified other than being beta-haemolytic organisms or group A streptococci. Limitations in ability to serotype the skin streptococci were clearly a problem in these investigations (Blumberg \& Feldman, 1962; McCullough et al., 1951).

In 1954, there occurred a significant epidemic of AGN at the Red Lake Indian Reservation in northern Minnesota. Two-thirds of the sixty-three patients reported to develop AGN were definitely considered to have pyoderma as the antecedent of that complication, with pharyngitis playing a minimal, if any, role in the outbreak (Kleinman, 1954). The epidemic peak of AGN at Red Lake occurred in late summer when weather in that particular area is hot and humid, not unlike that experienced in the south eastern area of the United States. The first outbreak of AGN at Red Lake is perhaps best remembered because the epidemic strain of streptococcus proved to be a new serotype-M-49 (Updyke, Moore \& Conroy, 1955) and was the first serotype of streptococcus found to be clearly related to AGN following skin infection. This strain, subsequently known as the Red Lake strain, has now been incriminated as a cause of skin infection and AGN in many parts of the world.

During the mid 1960s renewed interest in the role of skin infection and AGN developed largely as a result of extensive investigations in three particular areas: Alabama, Red Lake and Trinidad. It is 
apparent that certain findings have been common to each area. Each study has contributed distinct new information and collectively they have significantly furthered our understanding of the epidemiology of pyoderma and AGN. The more exciting observations include: the recognition of certain serotypes, including new or provisional M-types, to be of major importance in AGN; further documentation of 'impetigo streptococci' as a distinct group of strains in which serotype and site of infection appear clearly related; the demonstration that streptococcal antibody responses in skin and respiratory infection clearly differ and some interesting studies of subclinical nephritis that have implication with regard to the pathogenesis of AGN. In terms of numbers of cases of AGN, the Alabama studies have revealed a consistent number of cases to occur on an endemic basis within a given population each year; limited cases of AGN occurred at Red Lake, with a second epidemic occurring in 1966 and interestingly it, too, was associated with type 49 streptococci (Anthony et al., 1967). In Trinidad there have been massive epidemic waves of AGN. Type 49 streptococci and at least two new serotypes have been incriminated in these epidemics (Parker, 1969).

Some of the observations and results of studies at Alabama are being reported in detail here. These results and certain of those from Red Lake and Trinidad are compared. Individual or unique contributions of studies from the three particular areas will be emphasized.

\section{Characteristics of streptococcal impetigo}

Streptococcal impetigo lesions characteristically begin as small vesicles, becoming purulent and finally developing thickened honey-coloured crusts. A typical example of the latter type lesions is illustrated (plate 2, between pp. 679 and 680). It is not unusual to see lesions in varying stages in a given patient, or among siblings within a given family. The streptococcal skin lesions seen among Indian children at Red Lake and among natives of Trinidad are remarkably similar to those illustrated here and lesions of this type clearly predominate in each area. Chronic indolent lesions ('ecthyma'), are also observed and collectively the various skin lesions are simply referred to as pyoderma. The clinical appear- ance of streptococcal impetigo is similar in patients with and without the complication of AGN.

The similarity of bacteriologic findings at Alabama, Red Lake and Trinidad, have also been remarkable (Dillon, 1968; Anthony, Perlman \& Wannamaker, 1967; Potter et al., 1968; Parker et al., 1968). Table 1 illustrates the frequency of recovery of group A streptococci, with or without staphylococci, from children seen during recent years in Alabama. It is to be noted that presence of staphylococci in such skin lesions does not alter their appearance. Indeed, staphylococci appear for the most part to play a secondary role. In our experience the presence of penicillin-resistant staphylococci in skin lesions has in no way interfered with effective treatment of streptococcal impetigo with penicillin (Dillon, 1970).

Limited numbers of patients with bullous impetigo are also seen in Alabama and it is in lesions of this type that the staphylococcus is of primary aetiologic significance (Dillon, 1968). Most of the bullous staphylococcal isolates are members of phage group II, usually type 71 , and may or may not be sensitive to penicillin. When such lesions become secondarily infected with streptococci, as has been observed to be the case among patients studied here, they assume the typical purulent appearance of streptococcal lesions. Infection that remains limited to staphylococci leads to formation of a very thin and varnishlike crust with a bit of serous exudate at the margin of the lesion; staphylococci may be isolated from this margin until the lesion is healed. There are other clinical differences observed among patients with streptococcal impetigo as opposed to bullous impetigo which the author has described in more detail elsewhere (Dillon, 1968). One of the principal differences, however, is the more pronounced association of lymphadenopathy with streptococcal impetigo.

\section{Epidemiologic features of streptococcal impetigo and acute glomerulonephritis}

The peak seasonal incidence of impetigo in the south-eastern area of the United States occurs during the hot and humid months of late summer and early autumn, and the seasonal pattern of AGN associated with impetigo parallels this pattern. Fig. 2 depicts the

TABLE 1. The recovery rate for group A streptococci, with or without concomitant isolation of staphylococci are shown for patients with non-bullous impetigo seen over a period of 3 years. Patients with bullous impetigo are shown for comparison

\begin{tabular}{lcccccc}
\hline Clinical type & $\begin{array}{c}\text { Number } \\
\text { of } \\
\text { patients }\end{array}$ & $\begin{array}{c}\text { Total } \\
\text { Group A } \\
\text { streptococci }\end{array}$ & $\begin{array}{c}\text { Pure } \\
\text { streptococci }\end{array}$ & $\begin{array}{c}\text { Streptococci } \\
+ \\
\text { staphylococci }\end{array}$ & $\begin{array}{c}\text { Pure } \\
\text { staphylococci }\end{array}$ & $\begin{array}{c}\text { No } \\
\text { growth }\end{array}$ \\
\hline $\begin{array}{l}\text { Non-bullous } \\
\text { Bullous }\end{array}$ & 878 & $92 \%$ & $34 \%$ & $66 \%$ & $4 \%$ & $4 \%$ \\
\hline
\end{tabular}




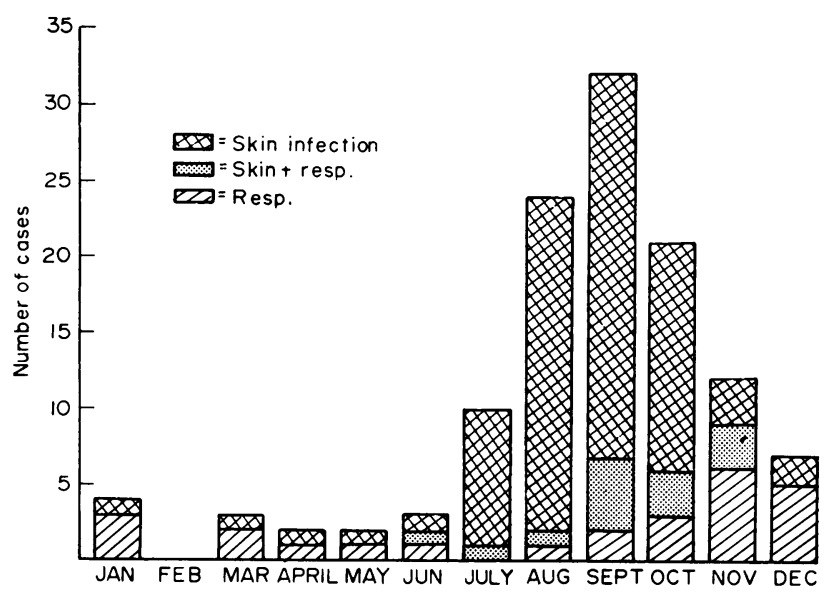

FIG. 2 Epidemiologic features of 120 cases of AGN, seen in Alabama over a period of 3 years are illustrated. The relation between season and primary antecedent site of infection reveals that skin infection is predominant, and peak months are late summer and early autumn.

experience in Alabama over a 3-year period (196466) when altogether 120 children with AGN were studied. It is apparent from this figure that the skin is the principal site of antecedent infection of AGN seen in this particular population. August and September are annually peak months for AGN following skin infection. The seasonal pattern of epidemic AGN at Red Lake is noteworthy in that both the original and the second outbreak of AGN following pyoderma occurred during summer and early autumn months, following an almost identical pattern to the Alabama experience illustrated in this figure.

In Trinidad, where massive waves of AGN following skin infection have been reported, streptococcal impetigo appears to be common at all seasons of the year. The outbreaks of AGN have been nonseasonal, occurring in an irregular fashion (Parker, 1969). The lack of a definite seasonal pattern for skin infection and AGN in Trinidad is most likely explained by that tropical island's climate, which varies little year round. Non-seasonal, endemic or epidemic skin infection and AGN also occurs in other areas in the Caribbean, as well as in other parts of the world including Ceylon (personal communication from Dr Barry M. Panter). The role of humidity and/or a rainy season in contributing to upswings of skin infection and AGN has not been completely clarified but in general such conditions seem to favour their increase. Such climatic factors may create conditions favourable for breeding of biting insects, such as the mosquito, which contribute to an increased incidence of minor skin trauma that in turn predisposes to streptococcal skin infection.

We know less about the mode of transmission of streptococcal impetigo than that of streptococcal respiratory infections. In the latter, direct spread occurs via the respiratory tract; factors of importance include active infection, quantity of organisms shed and duration of the carrier state (Wannamaker, 1954). Among the factors currently considered to contribute to the development of or spread of impetigo are: overcrowding, poor skin hygiene, an increased incidence of minor skin trauma and inadequate health-care facilities resulting in neglect of skin infection, permitting persistence of organisms which can then infect other contacts. The indolent nature of impetigo may further contribute to such neglect. Direct contact with lesions, hand contamination, and insects that serve as mechanical vectors in transporting viable organisms may all play a role in the spread of impetigo. In support of the latter, are interesting observations from Trinidad that the Hippelates fly, a small gnat-like insect, is capable of carrying viable organisms from patient to patient. These insects feed upon skin sores and are thought to play a role in the passive transfer of streptococci from patient to patient (Bassett, 1967; Parker, 1969).

Recent interesting studies from Red Lake (Dudding et al., 1970) suggest that streptococci associated with impetigo may reside on the intact skin of patients prior to the development of skin lesions. It appears that patients with impetigo might transmit organisms to siblings or other intimate contacts and that streptococci peculiar to skin infection have the capability of survival on normal skin for days or weeks prior to the actual development of lesions. Colonization of 'healthy' skin also preceded the recovery of streptococci from the respiratory tract when the latter was found to be colonized. 
Such observations offer intriguing evidence of possible important differences in streptococci causing skin and respiratory infection.

Patients with streptococcal impetigo not infrequently harbour organisms in the upper respiratory tract. In our own experience these organisms are commonly of the same serotype as that found in the skin lesions, being serotypes related to impetigo rather than pharyngitis (Dillon et al., 1967). The concomitant isolation of streptococci from skin and respiratory sites in patients with impetigo (including those with AGN) studied in Alabama has averaged approximately $30 \%$ during the different years of study. Typically, small numbers of organisms are found in the respiratory tract. Acute infection of the respiratory tract is uncommon, and it seems unlikely that colonization of the respiratory tract plays a major role in the spread of impetigo (Dillon, 1968). However, among individual patients or families, the respiratory tract may serve as a transient reservoir for organisms capable of infecting the skin. The Trinidad and Red Lake experiences with regard to the simultaneous recovery of streptococci from the skin and respiratory tract have been similar to that in Alabama, and there is general agreement that the respiratory tract is likely colonized secondarily from skin infection rather than vice versa. This concept is strongly supported by the detailed observations prior to and during the second outbreak of nephritis at Red Lake (Anthony et al., 1969).

Returning to Fig. 2, certain patients included in this figure are shown as having both skin and respiratory infection; skin infection was the predominant or major site of antecedent infection in this group. In most of these patients, respiratory symptoms were minor and could not be correlated with the recovery of streptococci from the respiratory tract. It is further apparent from this figure that when cases of AGN have followed throat infection only, the typical seasonal pattern of respiratory infection, namely, autumn, winter or early spring months has been observed. In contrast to the group of patients just mentioned with skin infection and minor respiratory symptoms, the latter group of children have uniformly had a primary complaint of sore throat, and occasionally scarlet fever, in the absence of any superficial skin infection.

Although the majority of patients in these recent studies are members of darker skin races, it is not possible at present to state clearly that there is a racial predeliction for skin infection. Socio-economic factors might account for this aspect of the problem. Age is of interest in that there is a definite predeliction for younger children to have streptococcal skin infection in contrast to pharyngitis, which is more common in early school years. It is not unusual to encounter streptococcal impetigo in infants and young children. A high proportion of those children studied in Alabama include pre-school children, the mean age being just under 6 years. It has not been unusual to see children under 3 years of age with AGN following impetigo.

Characteristics of streptococci associated with skin infection and acute glomerulonephritis

Serological typing of skin streptococci

A brief description of the methods of serotyping of streptococci is necessary in order to understand the rather difficult problems that have been encountered in the proper identification of those organisms isolated from skin lesions. Current knowledge of the epidemiology of acute glomerulonephritis associated with impetigo has resulted from extensive efforts of several laboratories to identify streptococci causing skin infections.

Group A streptococci are serotyped by two methods, the Griffith slide agglutination method (Griffith, 1934) and the precipitin method of Swift, Wilson \& Lancefield (1943), which identifies the $\mathrm{M}$-antigen of the streptococcus. The T-antigens of group A streptococci are serotyped by the agglutination method, employing trypsinized suspensions of whole streptococcal cells. T-antigens are cell-wall proteins but their role in streptococcal infection remains uncertain. Their principal usefulness is in epidemiologic studies. The Griffith method has been used for many years in major reference laboratories. It is possible to identify over $90 \%$ of strains of group A streptococci regardless of their source of isolation by this method. The principal disadvantage is a relative lack of specificity, particularly for certain strains common in skin infections, since these strains often react with more than one $\mathrm{T}$-antiserum resulting in agglutination patterns or complexes.

M-antigens of group A streptococci are responsible for virulence and since they are type-specific (it is rare for a strain to possess more than one Mantigen), the identification of the M-antigen is a highly useful serologic-epidemiologic tool. Protective antibody to $\mathbf{M}$-antigen develops in the infected host, and type-specific immunity to a particular serotype of streptococcus can be determined. The primary disadvantage of the serotyping of streptococci by the M-precipitin method alone is that it is not uncommon to encounter upwards of $50 \%$ of the strains in any large collection of streptococci which cannot be identified with available M-antisera. Indeed, this has been a chronic problem in studies of skin infection. It is presently recognized that the dual system of $M$ and T-typing provides clear advantages to the investigator in that strains which might not otherwise be identified can be initially characterized by T-typing; M-typing often becomes a simpler and a less time consuming procedure, since $\mathrm{T}$-antigens 
or complexes can generally be related to certain Mserotypes, limiting the number of $\mathbf{M}$-antisera required for initial testing of the strain in question.

\section{Impetigo streptococci}

The observations of Parker and colleagues that limited strains of streptococci were commonly isolated from skin lesions but seldom from other forms of infection first drew attention to the possibility that the biologic characteristics of skin streptococci might somehow differ from those strains isolated from other sources (Parker et al., 1955). A relationship between the serotype of the streptococcus and the site of infection was thus postulated and these investigators coined the term 'impetigo streptococci'. In the original studies, few of the impetigo streptococci could be identified with available Mantisera and it was suggested that these skin strains either lacked M-antigen, or were representative of strains for which antisera was difficult to prepare, or were perhaps representative of new serotypes. We now know that all three of these postulates were true. There are among skin streptococci strains which are representative of serotypes for which Mantisera are difficult to produce; there are others that seem to be weak in their production of M-antigen or appear to have lost $M$-antigen; and finally, there are now several examples of distinct new M-serotypes which have been described as a result of epidemiologic studies of skin infection and AGN. It has become increasingly apparent that streptococci most often associated with skin infection are a rich source of new M-types.

Table 2 contains a list of streptococci now recognized to be clearly associated with skin infection. T-agglutination patterns are shown and $M$ serotypes which share these particular T-antigens or agglutination complexes are indicated as being found either predominantly or infrequently among skin strains. The first three agglutination complexes noted in this particular table, 3/13/B3264, $8 / 25 /$ Imp.19, and $5 / 27 / 44$, were those originally reported to be 'impetigo streptococci'. It has become apparent (from studies in Alabama, at Red Lake, Trinidad and elsewhere) that the first two agglutination complexes illustrated are particularly important in skin infection wherever such infections have been studied. In addition, it is now recognized that certain other group A streptococcal serotypes appear to be commonly involved in skin infections but are less important or infrequently found in other forms of infection. This includes certain strains which can be identified by agglutination as T-type 11 or 12 , often non-M-typable with available reference sera, but some of which are representative of provisional new types of streptococci (see below); strains agglutinated as T-14/49 which are M-type 49; strains which are agglutinated as T-4 and may be members of provisional M-type 60; and strains of the agglutination complex 5/17/19/23/47 among which the new Mserotype 54 was found. The relationship of certain of these serotypes of streptococci to AGN following pyoderma is described in detail shortly. It is pertinent to point out here that of the last ten M-serotypes of streptococci to be recognized as new or provisional new serotypes, all have been found as a result of studies of skin infection. These include Mtypes 52, 53, and 54, from Red Lake (Top et al., 1967); type 55 (Potter et al., 1968), type 57 (Parker et al., 1968), provisional type 58 (personal communication from Dr W. R. Maxted) from Trinidad; provisional types 59, 60 and 61 identified in our population in Alabama (Dillon \& Avery-Reeves, manuscript in preparation). Type 56 was isolated from a patient with pyoderma in Tennessee (Johnson et al., 1968).

TABLE 2. Serotypes of streptococci predominantly associated with skin infection are shown. M-types found among the prevalent T-agglutination complexes common to impetigo streptococci are listed. M-types with similar T-types, but infrequently associated with skin infection, are shown for comparison

\begin{tabular}{|c|c|c|}
\hline \multirow[b]{2}{*}{ T-agglutination } & \multicolumn{2}{|l|}{ M-serotypes } \\
\hline & Predominantly skin & Rarely skin \\
\hline $3 / 13 / B 3264^{* *}$ & $33,39,41,43,52^{*}, 53^{*}$ & 3,13 \\
\hline 8/25/Imp.19** & $\begin{array}{l}2,55^{*}, 57^{*} \text {, Prov. } 58^{*} \\
\text { (occ. } 8 \text { and } 56)\end{array}$ & 25 \\
\hline $5 / 27 / 44$ (Originally $5 / 11 / 12 / 27 / 44$ )** & Usually non-typable & $5,27,44$ \\
\hline 11,12 , or $11 / 12$, or 'W' Pool & $\begin{array}{l}\text { Usually non-typable } \\
\text { Occasionally Prov. 59*, 61*, M-11 }\end{array}$ & $11,12,22$ \\
\hline $14 / 49$ & 49 & 14 \\
\hline 4 & $\begin{array}{l}\text { Prov. } 60^{*} \text {, } \\
\text { Occasionally M-4 }\end{array}$ & \\
\hline $5 / 17 / 19 / 23 / 47$ & $54^{*}$ & \\
\hline
\end{tabular}

** Original agglutination pattern considered by Parker et al. to be 'impetigo streptococci'.

* New or provisional new M-serotypes. 
Streptococci found in the agglutination complex 3/13/B3264, the complex most commonly identified where impetigo lesions are observed, include several different M-serotypes, not all of which are prevalent in a given area. Thus, in Alabama M-types 33, 39 and 52 have more often been observed whereas in Minnesota types 41,52 and 53 and more recently 43 have been found. Type 52, also found in Trinidad, is common in all three areas. Many strains in this complex remain unidentified as to M-type. Among the $8 / 25 /$ Imp.19 strains, those incriminated in endemic or epidemic AGN, most of which can now be identified by M-type, are discussed subsequently. The 8/25/Imp.19 strains from patients with uncomplicated impetigo seen in Alabama and at Red Lake are frequently non-M-typable. (M-types 8, 25 and 56 are occasionally found). Most of the strains within the agglutination complex 5/27/44 have been nonM-typable; they are found less frequently in impetigo than the above two complexes.

In Alabama, strains identified only as T-12 are commonly associated with impetigo but are rarely M-typable. (One of the provisional new serotypes, provisional type 59, has been observed among children with uncomplicated impetigo and this strain may show an agglutination reaction with either T-type 11,12, or both). M-type 54, on the basis of current epidemiologic information, is not widely distributed.

\section{The nephritogenic skin streptococci}

The serotypes of streptococci now recognized of importance in causing AGN following skin infection differ from those commonly associated with pharyngitis and AGN as clearly as pharyngitis itself differs from impetigo. This is truly one of the fascinating features of the epidemiology of AGN.

Table 3 summarizes the relation between serotypes of streptococci and the common site of infection of the currently recognized nephritogenic streptococci including certain provisional new types which appear of definite, though as yet limited importance. Nephritogenic strains associated with throat infection include types $1,3,4,12$ and 25, types 1 and 12 having been most often associated with epidemic AGN. In spite of vigorous efforts to identify strains of these particular types where nephritis has followed skin infection, they have been rarely found.

Current knowledge of the nephritogenic serotypes of streptococci associated with skin infection is largely based on the recent Alabama, Red Lake and Trinidad studies being discussed herein. The strains of principal importance are: M-2, M-55 and M-57, all of which share a common agglutination complex, $8 / 25 /$ Imp. 19 and M-49. The provisional new types, 59, 60 and 61, are further discussed below. Neither
TABLE 3. Serotypes of streptococci associated with AGN are listed, according to T- and Mantigens and the common site from which such types are isolated. The limited distribution of certain types is indicated

\begin{tabular}{llllc}
\hline \multicolumn{2}{c}{ Serotype } & & \multicolumn{2}{c}{ Infection site } \\
\cline { 1 - 2 } \cline { 5 - 5 } T-antigen & M-antigen & & Skin & Throat \\
\hline 1 & 1 & & + \\
$8 / 25 /$ Imp.19 & 2 & & + & + \\
$3 / 13 /$ B3264 & 3 & & & + \\
4 & 4 & & + \\
4 & Prov. 60* & & + \\
6 & 6 & & + \\
12 & 12 & & + \\
$8 / 25 /$ Imp.19 & 25 & & + \\
$14 / 49$ & 49 & & + & \\
$3 / 13 /$ B3264 & $52^{* *}$ & & + & \\
$8 / 25 /$ Imp.19 & 55 & & + & \\
$8 / 25 /$ Imp.19 & $56^{* * *}$ & & + & \\
$8 / 25 /$ Imp.19 & 57 & & + & \\
'W' Pool & Prov. 59* & + & \\
11 & Prov. 61* & + & \\
\hline
\end{tabular}

* Alabama Studies: Provisional 60 strongly implicated; prov. 59 and 61 limited cases.

** Limited cases, Red Lake; Alabama; Trinidad.

*** Limited cases, Tennessee.

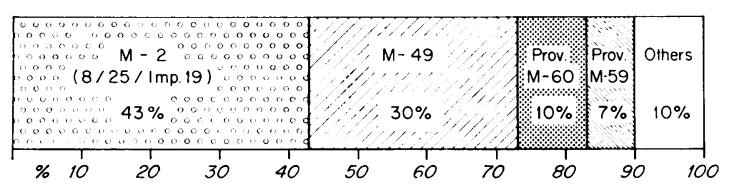

Fig. 3. Major nephritogenic serotypes of streptococci isolated from skin lesions of patients with AGN (149= $49 \%$ ) and their siblings $(155=51 \%)$ over a 5 -year period (1964-68) of study in Alabama are shown. M-type 2 has been most common. The total number of strains was 304 .

type 52 nor 56 appear of major importance in AGN at this time.

Type 49 streptococci, from an historical point of view, are of special interest since this was the first serotype to be clearly related to skin infection and AGN and remains the one type which has been clearly implicated as a significant cause of skin infection and AGN in many parts of the world. Its distribution includes widely different areas in the United States, Europe and Trinidad where it has been a major epidemic strain. The author has received type 49 strains isolated from children in Ceylon reported to have AGN. Unlike the other strains presently associated with skin infection and nephritis, type 49 has also been implicated in AGN following pharyngitis, but is most often associated with pyoderma.

M-type 2 streptococci have been the major cause of AGN in patients studied in Alabama (Dillon, Maxted \& Reeves, 1968). As shown in Fig. 3, this serotype accounted for nearly half the cases of AGN 
from which the skin isolates were identified during the first 5 years of investigation. It is annually the most prevalent type among the AGN patients and their siblings; of thirty patients with skin infection and AGN seen in 1969, two thirds had M-2 infection.

These type M-2 strains causing skin infection and AGN differ from 'classical' M-2 streptococci found in throat infection in their agglutination reactions. The latter possess the T-type 2 antigen only; the type 2 skin strains agglutinate only with T-antisera 8/25/Imp.19. In this regard, they resemble M-types 55 and 57, two of the major nephritogenic strains isolated in Trinidad.

M-2 streptococci with the agglutination complex $8 / 25 /$ Imp. 19 have been isolated from the pharynx of some patients with type 2 skin infection, but in no case have we identified them in patients with pharyngitis and AGN. Even more impressive, however, is the infrequency with which they have been isolated from patients other than in families where one or more cases of AGN have been identified.

Other types of importance in AGN in Alabama are $M-49$, the second most common type we find, and the provisional types 60 and 59. Provisional type 60 , one of three new provisional types identified in the Alabama studies (Dillon \& Reeves, in preparation) have been isolated annually from patients with AGN in this population. This strain is agglutinated with T-4 antisera, as is M-type 4. The M-antigens (4 and prov. 60) do not cross-react, however, and we have not found M-4 strains to be related to pyoderma and AGN. Provisional type 60, like M-2, has been infrequently found other than in families in which AGN has occurred. Provisional type 59 has caused sporadic cases of AGN, and during 1 year was fairly commonly isolated from patients with 'uncomplicated impetigo'. Provisional type 61 is the least common of the three new types; the future role of these strains as a major cause of AGN remains to be determined. Their identification, however, has served to further clarify the epidemiology of skin infection and AGN.

It has been previously mentioned that many of the impetigo streptococci have been difficult to identify other than by T-agglutination. By employing currently available antisera, plus that for provisional new types, we have been able to identify over $95 \%$ of the streptococcal isolates from skin lesions of patients with AGN. The majority of nephritogenic strains at Red Lake have been M-type 49. In the Trinidad studies the preparation of antisera for the new types 55 and 57 made their identification possible. There is no longer doubt that the nephritogenic streptococci from skin lesions possess M-antigens.

There remain among the impetigo streptococci many strains which as yet are classified only by
T-agglutination typing. Experience has shown, however, that new serotypes are to be found among such strains. It is increasingly apparent that the skin streptococci will require $\mathbf{M}$-antisera prepared against appropriately selected wild strains causing skin infection, if maximum success in identifying $\mathbf{M}$ antigen is to be achieved.

\section{Characteristics of acute glomerulonephritis associated with skin infection}

The clinical picture of AGN associated with pyoderma is identical to that seen with streptococcal pharyngitis. The classical manifestations of hypertension, oedema, and typical urine findings prevail regardless of the antecedent site of infection. All degrees of severity of AGN have been observed in children with pyoderma including some who have presented with acute encephalopathy, and others presenting with acute congestive heart failure. The majority have presented with an acute illness characterized by a variable degree of hypertension and oedema (Dillon et al., 1967). The observed sex ratio in patients with AGN has been approximately $60 \%$ males to $40 \%$ females until 1969 , during which time there was a 2 to 1 male to female ratio. In contrast, there is no difference in sex incidence of children with 'uncomplicated impetigo'. This prevalence of males in the AGN population remains unexplained. The average hospital stay for children with overt evidence of AGN has been 10 days. Extended posthospital follow-up studies have revealed that microscopic haematuria persists in some instances for many months. In the majority of patients however, clinical and iaboratory parameters have returned to normal within a matter of weeks following the onset of the disease.

Although we have not performed kidney biopsies. they were obtained from children studied during the 1966 Red Lake epidemic and in Trinidad (personal communication from Dr B. F. Anthony and Dr Elizabeth Potter). The histopathological studies were essentially the same as those observed previously in children whose AGN followed pharyngitis. It thus appears from both the clinical and pathological standpoint that there is no difference in the disease with regard to the antecedent site of infection.

\section{Complement in clinical and subclinical nephritis}

The determination of beta 1-A/beta 1-C-globulin ( $\mathrm{C}^{13}$ component of complement) has proven of particular value in our studies of AGN following skin infection. Immunoelectrophoretic and radial immunodiffusion techniques have been used, the latter being preferable because of simplicity and the ease with which results can be quantitated (Derrick, Reeves \& Dillon, 1970). Complement is low during the acute phase of pcst-streptccoccal nephritis, but 
returns to normal within a few weeks of onset of the disease.

Many children with clinically overt AGN admitted to our studies have had both clinical and laboratory evidence, including low complement, of AGN when first examined. Additional cases of overt and subclinical AGN have been identified among sibling contacts of such index cases. Observations in the latter group have been of particular interest. Quantitative results of beta 1-C/beta 1-A-globulin levels in children with overt AGN, their siblings and a selected group of patients with 'uncomplicated impetigo' seen during one particular year are shown in Fig. 4.

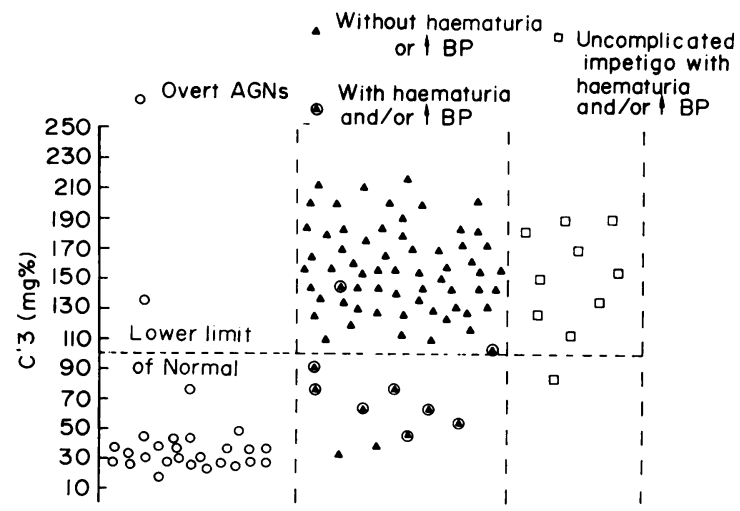

FIG. 4. This figure illustrates beta 1-C/beta 1-A-globulin determinations found in the three major clinical categories of patients studied with skin infection: AGN; siblings of AGN and 'uncomplicated impetigo'. Only ten of 255 patients in the latter group are illustrated. None of the remaining $\mathbf{2 4 5}$ children had levels lower than those shown here, and only $1 / 255$ had a value less than normal.

Serial determinations (three or more) were done in all patients admitted to that study. Initial or lowest levels are shown in the figure. The lower limit of normal is $100 \mathrm{mg} / 100 \mathrm{ml}$, and all but one patient with clinically overt evidence of AGN fell well below this level. The latter patient was seen relatively late during the course of AGN. As just described, siblings of patients with overt AGN are commonly infected with streptococcal serotypes identical to that isolated from the index case of AGN in the family. Siblings shown here most often had infection with either M-2 or M-49 streptococci, both prevalent nephritogenic strains that year. Among the seventytwo AGN siblings nine had a low beta $1-\mathrm{C} /$ beta 1-A-globulin, including two in whom this was the only evidence of subclinical AGN. In several other siblings, the drop in complement preceded the occurrence of haematuria. This relation of haematuria and low complement is further discussed below.

A total of 255 patients with 'uncomplicated impetigo' had serial complement determinations but for clarity only those ten individuals found to have either microscopic haematuria or a transient elevation of blood pressure are depicted in this particular figure, since none of the remaining 245 patients had complement levels less than these ten. Children with 'uncomplicated impetigo' are typically infected with strains infrequently associated with nephritis; identical serotypes are usually to be found among siblings within a given family. The one patient in this group of 255 children with 'uncomplicated impetigo' found to have a low beta 1-C/beta 1-Aglobulin, however, was an only child who was infected with a nephritogenic streptococcus. He was also the only patient in the 'uncomplicated impetigo' group found to have both microscopic haematuria and a transient elevation of blood pressure.

It is difficult to be certain that transient microscopic haematuria, or a single elevation of blood pressure is of significance in evaluating patients with streptococcal skin infection. As a result of observations now extending over a 3-year period, we have found that beta $1-\mathrm{C} /$ beta $1-\mathrm{A}$ determinations are particularly sensitive in detecting laboratory evidence of subclinical AGN. A total of 198 siblings of patients with overt AGN have been screened. A summary of abnormalities in the eighteen children we considered to have evidence of subclinical AGN is shown in Table 4. The nephritogenic serotype causing infection in both index case and affected sibling is included. Seventeen of the eighteen had a low beta $1-\mathrm{C} /$ beta 1-A-globulin demonstrated during the course of infection.

The most striking observation however, was the demonstration of a low beta 1-C/beta 1-A-globulin level whether or not haematuria occurred. Low

TABLE 4. The finding of low beta 1-C/beta 1-A-globulin, with or without other abnormalities, in siblings of patients with clinically overt AGN is shown. Nephritogenic serotypes are included, and reveal the predominance of $\mathrm{M}-2$

\begin{tabular}{ccccc}
\hline $\begin{array}{c}\text { Streptococcal } \\
\text { serotype }\end{array}$ & $\begin{array}{c}\text { Number of } \\
\text { patients }\end{array}$ & Low C C $^{\mathbf{3}}$ & Haematuria & $\begin{array}{c}\text { Elevated } \\
\text { blood pressure }\end{array}$ \\
\hline Type 2 & 14 & 13 & 7 & 4 \\
Type 49 & 2 & 2 & 0 & 1 \\
Prov. 60 & 2 & 2 & 1 & 0 \\
Totals & 18 & $17 / 18$ & $8 / 18$ & $5 / 18$ \\
\hline
\end{tabular}


complement occurred in the absence of haematuria in ten of seventeen patients, and preceded haematuria in four of the remaining patients. Complement remained normal in two of the eight siblings with haematuria and one of the five with transient hypertension. The combination of low complement, microscopic haematuria, and transient hypertension, occurred together in only one of the eighteen siblings. None of this particular group progressed to develop clinically overt AGN. The fact that changes in complement occurred prior to the development of microscopic haematuria, or in its absence, fits the current concept that an immunologic mechanism presumably induced by infection with a nephritogenic streptococcus, and involving utilization of complement, is responsible for the renal injury observed in AGN.

In contrast to these exciting observations in the siblings of children with overt AGN, among that large group of children with 'uncomplicated impetigo' studied during the same period of years (nearly 500 children) only fourteen had any of the above mentioned abnormalities. Only $3 / 14$ had a drop in complement, and in each case they were found to be infected with a recognized nephritogenic streptococcus prevalent in this population. Two of the three children with low complement also had microscopic haematuria. Only one of the three had all three of the abnormalities (the patient illustrated previously in Fig. 4). It is thus apparent that in a majority of children with 'uncomplicated impetigo' microscopic haematuria is infrequently observed and not often associated with or preceded by changes in complement. This observation is in keeping with earlier studies (Stetson et al., 1955) which demonstrated a higher incidence of haematuria in military recruits infected with a recognized nephritogenic strain of streptococcus than in those infected with serotypes not commonly associated with AGN.

We have concluded that beta 1-C/beta 1-Aglobulin determinations are particularly useful in confirming cases of clinically overt post-streptococcal AGN, in detecting subclinical cases of AGN in children at risk for this complication (notably those infected with a recognized nephritogenic strain) and in excluding the likelihood of poststreptococcal AGN in children with uncomplicated streptococcal skin infections caused by a variety of streptococci which infrequently caused AGN.

It has long been recognized that multiple cases of AGN occur within families within a short period of time of one another. We have documented four cases of post-streptococcal AGN following type 2 streptococcal skin infection in one family and three cases of overt AGN in two other families and several examples of two or more cases of clinical and/or subclinical AGN occurring within a given family. These observations further emphasize the necessity for prompt and early studies of siblings of children with overt AGN for either detection of or possible prevention of further cases.

\section{Streptococcal antibody responses-impetigo and nephritis}

The relation between streptococcal immune response and site of infection is also of particular interest in considering differences in skin and respiratory infection. Limited observations from earlier studies (Burnett, 1962; Markowitz et al., 1965) suggested a somewhat irregular or erratic ASO response in children with streptococcal impetigo. In reviewing these data, it is difficult to clearly relate bacteriologic to serologic findings; however, the pattern of poor ASO response in proven streptococcal impetigo was clearly suggested. We now know from the various epidemiologic studies that have been mentioned thus far that the nature of the streptococcal antibody response is clearly affected by the site of antecedent infection and in limited instances by the serotype of the infecting strain.

Anthony and co-workers (1967) found the antiDNAse-B test to be definitely superior to the ASO test in demonstrating serologic evidence of streptococcal infection in patients with pyoderma at Red Lake. In Alabama (Dillon \& Reeves, 1969) we have found that in patients with streptococcal impetigo, whether or not AGN complicates the infection, anti-DNAse-B titres are approximately twice as often elevated as ASO titres. Anti-DNAse-B titres have been found to be strikingly elevated in patients with skin infection and AGN in this population (Derrick et al., 1970); thus both the frequency of and magnitude of immune response is greater to DNAse$B$ antigen than streptolysin $O$. An extensive study comparing differences in antistreptolysin $\mathrm{O}$ and anti-DNAse-B titres in patients with impetigo, AGN and siblings of AGN, has recently been completed and will be reported in detail (Dillon \& Reeves, in preparation). Certain findings of particular interest with regard to nephritis are shown in Fig. 5. (These antibody determinations were done in that same group of patients illustrated earlier in Fig. 2 in which the seasonal pattern of AGN in this population was demonstrated.) These patients, seen over a 3-year period, represented the first group with AGN in whom both anti-streptolysin O and anti-DNAse-B determinations were performed on one or more sera during the course of AGN. Findings in succeeding years have been quite similar to those illustrated here. Approximately $85 \%$ of the patients included in Fig. 5 had AGN following impetigo. Considering first anti-DNAse-B titres, the upper limit of normal in our population for children over 2 years of age is approximately 500, but under 2 nearer 90 . Among 


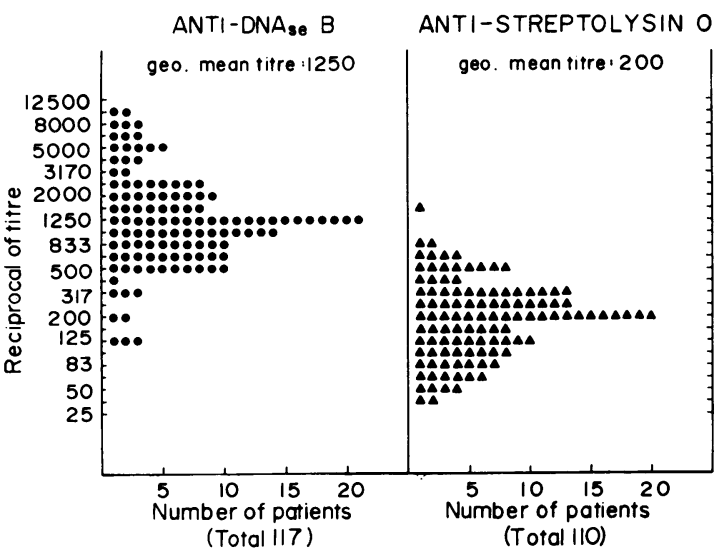

FIG. 5. A comparison of two streptococcal antibody tests-anti-DNAse-B and ASO-in children with AGN is shown. (This group of patients same as illustrated in Fig. 2.) Anti-DNAse-B titres are both higher and more frequently elevated than ASO titres in patients with skin infection.

those children illustrated here with titres less than 500 , were several under 2 years of age; of the total number of children with skin infection and AGN $90 \%$ had an elevated anti-DNAse-B titre. The mean anti-DNAse-B titre of 1250 is quite impressive and reflects the vigorous response seen particularly in patients with skin infection and AGN. Included in this figure are a limited number of patients whose primary antecedent site of infection was the throat. Although anti-DNAse-B titres were elevated in $80 \%$ of that group, the titres were less often as strikingly elevated as in patients with skin infection. Considering the ASO titres (seven fewer patients had ASO titres done) the upper limit of normal for children of 2 years of age and over was 125; that for children under 2 approximately 80 . It is clear that titres of many with AGN were not elevated. Among the children with AGN following skin infection, only $50 \%$ had an elevated ASO titre. Highest ASO titres were found among the small group of children studied with respiratory infection and AGN. The titres were also more frequently elevated in that group $(80 \%)$ than in those with skin infection $(50 \%)$. The relatively low mean ASO titre (200) in AGN patients reflects the dominance of skin infection as the antecedent site in this population.

We have concluded that the anti-DNAse-B is a superior streptococcal test in the study of children with skin infection and AGN, and indeed, consider it the primary streptococcal antibody test for this population or others where streptococcal skin infection is predominant. We consider the ASO test less reliable as a means of serologic documentation in children with skin infection and AGN, since the finding of a normal ASO titre in such patients could clearly be misleading.
In studies of AGN and skin infection in Trinidad the antihyaluronidase has been used as a second test along with the ASO test for serologic studies. It has been demonstrated there that the antihyaluronidase is a more efficient streptococcal antibody test than is the ASO test (Potter et al., 1968). It is thus apparent that in the three widely different areas where extensive studies of skin infection and AGN have recently been done, anti-streptolysin $O$ patterns are similar in each area and a second antibody test has been found necessary to afford maximum serologic definition of streptococcal infection.

The anti-NADase (formerly called anti-DPNase) streptococcal antibody test was previously found to be of value in studies of AGN following respiratory infection. It is now apparent that this test is unreliable in studies of streptococcal skin infection and AGN (Wannamaker, 1970).

The relatively feeble anti-streptolysin $\mathrm{O}$ responses in streptococcal skin infections are not completely explained. Several explanations present themselves for consideration. Streptolysin $O$ production or its antigenicity is conceivably inhibited to some degree when streptococci are growing in cutaneous sites. Serotypes commonly producing impetigo may be poor producers of streptolysin $\mathrm{O}$. In any event, present evidence suggests that the marked differences observed in ASO and anti-DNAse-B responses in patients with streptococcal skin infection as opposed to respiratory infection are a function of the site of infection.

\section{Treatment of streptococcal infections}

It is not intended here to discuss at length the appropriate therapy of streptococcal pyoderma, but the question commonly arises as to whether or not the treatment of streptococcal skin infection can adequately prevent the development of AGN. It seems pertinent to emphasize several key points. First, there is the common question regarding the relative merits of topical therapy versus systemic therapy for streptococcal impetigo. In our recent studies (Dillon, 1970) we have been able to clearly demonstrate the marked superiority of parenteral versus topical therapy in eradicating streptococci, rapidly clearing lesions clinically, eliminating organisms also present in the upper respiratory tract. More recently (Derrick \& Dillon, 1970), we have shown that either penicillin or erythromycin administered orally in appropriate doses is quite effective in prompt bateriologic and clinical clearing of skin infection. The advantages of such therapy over topical therapy have been thoroughly discussed. The occasional patient with one or two discrete lesions (particularly children whose infection is brought to the attention of a physician quite early) can possibly be treated safely with topical therapy. 
Our own experience, and that of others studying populations where the economicaliy disadvantaged group of patients is seen, suggests that in cases where impetigo is fairly extensive and where lesions are commonly present for days or weeks prior to seeking attention, topical therapy is impracticable, and indeed, an unwise choice.

In spite of vigorous efforts to do so, we have not been able to prove unequivocally that we can prevent cases of AGN by prompt treatment of streptococcal skin infection. We do feel, however, that prompt treatment of patients with skin infections with a recognized nephritogenic strain is a most important public health measure in preventing further spread of these organisms. This is yet another reason to pursue family studies of patients with skin infection where an index case of AGN has occurred. Prompt therapy in infected sibling contacts, if infection has not been present too long, might prevent $A G N$ in this 'high risk' group.

\section{Acknowledgments}

This work was done under the sponsorship of the Commission on Streptococcal and Staphylococcal Diseases of the Armed Forces Epidemiological Board, and was supported in part by the U.S. Army Medical Research \& Development Command, Department of the Army, under research contract No. DA-49-193-MD-2635. Additional support was granted by Ross and Wyeth Laboratories.

\section{References}

Anthony, B.F., Kaplan, E.L., Chapman, S.S., Quie, P.G. \& Wannamaker, L.W. (1967) Epidemic acute nephritis with reappearance of type 49 streptococcus. Lancet, ii, 787.

Anthony, B.F., Perlman, L.V. \& Wannamaker, L.W. (1967) Skin infections and acute nephritis in American Indian children. Pediatrics, 39, 263.

ANTHONy, B.F., Kaplan, E.L., Wannamaker, L.W., Briese, F.W. \& Chapman, S.S. (1969) Attack rates of acute nephritis following type 49 streptococcal infection of the skin and of the respiratory tract. Journal of Clinical Investigation, 48, 1697.

BassetT, B.C.G. (1967) Hippelates flies in acute nephritis. Lancet, i, 503.

BlumberG, R.W. \& Feldman, D.B. (1962) Observations on acute glomerulonephritis associated with impetigo. Journal of Pediatrics, 60, 677.

BurnetT, J.W. (1962) Management of pyogenic cutaneous infections. New England Journal of Medicine, 266, 164.

Derrick, C. Warren, Reeves, Mary S. \& Dillon, Hugh C. (1970) Complement in overt and asymptomatic nephritis after skin infection. Journal of Clinical Investigation, 49, 1178.

Derrick, C. Warren \& Dillon, H.C., JR (1970) Further studies on the treatment of streptococcal skin infection. Journal of Pediatrics. (In press).

Dillon, H.C. (1967) Pyoderma and nephritis. Annual Review of Medicine, 18, 207.

Dillon, H.C. (1968) Impetigo contagiosa: Suppurative and non-suppurative complications. American Journal of Diseases of Children, 115, 530,

Dillon, H.C. (1970) The treatment of streptococcal skin infections. Journal of Pediatrics, 76, 676.

Dillon, H.C., Moody, M.D., Maxted, W.R. \& Parker, M.T. (1967) The epidemiology of impetigo and acute glomerulonephritis-results of serological typing of group A streptococci. American Journal of Epidemiology, 86, 710.

Dillon, H.C., Maxted, W.R. \& Reeves, M.S. (1968) Acute glomerulonephritis following skin infection due to streptococci of M-type 2. Lancet, i, 543.

Dillon, H.C. \& Reeves, M.S. (1969) Streptococcal antibody titers in skin infection with AGN. Pediatric Research, (Abstract), 3, 362.

Dudding, B.A., Burnett, J.W., Chapman, S.S. \& WannaMAKER, L.W. (1970) The role of normal skin in the spread of streptococcal pyoderma. Journal of Hygiene, 68, 19.

Griffith, F. (1934) Serologic classification of Str. Pyogenes، Journal of Hygiene, 34, 542.

Johnson, J.C., Baskin, Reed C., Beachey, Edwin H. \& Stollerman, G.H. (1968) Virulence of skin strains of nephritogenic group A streptococci: New $M$ protein serotypes. Journal of Immunology, 101, 187.

Kleinman, H. (1954) Epidemic acute glomerulonephritis at Red Lake. Minnesota Medicine, 37, 479.

Markowitz, M., Bruton, H.D., Cuttner, A.G. \& Cluff, L.E. (1965) The bacteriologic findings of streptococcal immune response and renal complications in children with impetigo. Pediatrics, 35, 393.

McCullough, G.C., Coffee, J.Y., Trice, P.A., Stone, J.J. \& Crandall, H.L. (1951) Acute glomerulonephritis: Impetigo as an etiological factor. A review of 124 cases at Lloyd Noland Hospital, Fairfield, Alabama. Journal of Pediatrics, 38, 346.

Parker, M.T., Tomlinson, A.H.J. \& Williams, R.E.O. (1955) Impetigo contagiosa: The association of certain types of Staphylococcus aureus and Streptococcus pyogenes with superficial skin infections. Journal of Hygiene, 53, 458.

Parker, M.T. (1969) Streptococcal skin infection and acute glomerulonephritis. British Journal of Dermatology, 81, Suppl. 1, 37.

Parker, M.T., Bassett, D.C.J., Maxted, W.R. \& Arneaud, J.D. (1968) Acute glomerulonephritis in Trinidad: Serological typing of group A streptococci. Journal of Hygiene, 66, 657.

Potter, E. V., Siegel, A.C., Simon, N.M., McAninch, J., Earle, D.P., Poon-King, T., Mohammed, I. \& Abidh, S. (1968) Streptococcal infections and epidemic acute glomerulonephritis in South Trinidad. Journal of Pediatrics, 72, 871 .

Rammelkamp, C.H., JR, Denny, F.W., JR \& Wannamaker, L.W. (1952) Studies on the epidemiology of rheumatic fever in the Armed Services. In Rheumatic Fever (Ed. by L. Thomas), p. 72. University of Minnesota Press, Minneapolis.

Rammelkamp, C.H., JR \& Weaver, R.S. (1953) Acute glomerulonephritis. The significance of the variations in the incidence of the disease. Journal of Clinical Investigation, 32, 345.

Stetson, C.A., Rammelkamp, C H, JR, Krause, R.M., Kohen, R.J. \& Perry, W.D. (1955) Epidemic acute nephritis: Studies on etiology, natural history and prevention. Medicine (Baltimore), 34, 431.

Swift, H.F., Wilson, A.T. \& Lancefield, R.C. (1943) Typing group A hemolytic streptococci by $M$ precipitin reactions in capillary pipettes. Journal of Experimental Medicine, 78, 127.

Top, F.H., JR, Wannamaker, L.W., Maxted, W.R. \& ANTHONY, B.F. (1967) M-antigens among group A streptococci isolated from skin lesions. Journal of Experimental Medicine, 126, 667.

Updyke, E.L., Moore, M.S. \& Conroy, E. (1955) Provisional new type of group A streptococci associated with nephritis. Science, 121, 171 . 
WANNAMAKER, L.W. (1970) Differences between streptococcal infections of the throat and of the skin. New England Journal of Medicine, 282, (Part I), 23, (Part II), 78.

WANNAMAKER, L.W. (1967) Epidemiology of acute glomerulonephritis. In Acute Glomerulonephritis, 17th Annual Sym- posium on the Kidney, National Kidney Foundation (Ed. by J. Metcoff), p. 39. Little, Brown, Boston.

WANNAMAKER, L.W. (1954) The epidemiology of streptococcal infections. In Streptococcal Infections (Ed. by $\mathbf{M}$. McCarty), p. 157. University Press, Columbia. 